\title{
Le remodelage d'un organisme de formation par une politique volontariste d'utilisation des TICE
}

Le cas de l'organisation de la formation professionnelle dans la Marine Nationale

Ludovic Sar

\section{OpenEdition}

Journals

Édition électronique

URL : http://journals.openedition.org/communicationorganisation/4651

DOI : 10.4000/communicationorganisation.4651

ISSN : 1775-3546

Éditeur

Presses universitaires de Bordeaux

Édition imprimée

Date de publication : 1 juin 2014

Pagination : 283-296

ISBN : 978-2-86781-904-9

ISSN : $1168-5549$

Référence électronique

Ludovic Sar, «Le remodelage d'un organisme de formation par une politique volontariste d'utilisation des TICE », Communication et organisation [En ligne], 45 | 2014, mis en ligne le 01 juin 2017, consulté le 20 avril 2019. URL : http://journals.openedition.org/communicationorganisation/4651 ; DOI :

10.4000/communicationorganisation.4651 


\title{
Le remodelage d'un organisme de formation par une politique volontariste d'utilisation des TICE le cas de l'organisation de la formation professionnelle dans la Marine Nationale
}

\author{
Ludovic Sor ${ }^{1}$
}

\section{Introduction}

Professeur de sciences et techniques industrielles au Centre d'Instruction Naval (CIN) ${ }^{2}$ de Saint-Mandrier, le plus important organisme de formation professionnelle des armées françaises ${ }^{3}$, nous sommes acteur et observateur de la mise en place progressive d'une réforme de la formation initiée par la Marine Nationale à la fin des années 1990, réforme dont l'un des volets majeurs est une politique volontariste de recours aux TICE. Nous nous proposons d'évaluer l'impact de cette politique sur l'organisation de la formation, essentiellement dans le modèle pédagogique du CIN. Notre activité de chercheur étant, en l'occurrence, quasi déconnectée de notre activité professionnelle, notre analyse relève de la "double-posture » qui est celle du "praticien-chercheur» (Mias, 2003) : il s'agit d'une recherche-action distanciée.

\section{La problématique de la formation professionnelle dans la Marine Nationale}

La formation professionnelle dispensée par la Marine Nationale est soumise à des contraintes fortes.

1 Ludovic Sar, professeur certifié de sciences industrielles de l'ingénieur (option informatique et numérique), détaché au Centre d'Instruction Navale (CIN) de Saint-Mandrier, titulaire d'une maîtrise EEA (électronique, électrotechnique, automatique), d'un Master II et d'un doctorat en sciences de l'information et de la communication de l'Université du Sud Toulon-Var, chercheur associé au laboratoire I3m (Université du Sud Toulon-Var) ; ludovic1.sar@gmail.com

2 Dans la suite de cette étude, l'acronyme CIN sans stipulation fera référence au CIN de Saint-Mandrier.

3 Le CIN, qui accueille 8000 élèves et stagiaires et dispense 170000 heures d'enseignement par an, est :

- pôle d'enseignement technique et opérationnel pour l'ensemble du personnel de la Marine Nationale ;

- centre d'incorporation et de formation initiale ;

- pilote de la formation au management et à l'exercice de l'autorité pour les officiers mariniers ;

- pôle d'expertise pédagogique et pilote de la formation des formateurs ;

- pôle d'expertise pour les TICE;

- organisme certificateur pour la délivrance des titres inscrits au répertoire national des certifications professionnelles (RNCP). 


\section{$\mathrm{C} \& O \mathrm{n}^{\circ} 45$}

Tout d'abord, pour effectuer ses missions - connaître et anticiper, prévenir, dissuader, protéger, intervenir ${ }^{4}$ - elle doit être polyvalente. Aussi a-t-elle besoin de compétences multiples dans des domaines techniques complexes, concernant des générations différentes de bâtiments et de matériels. Comme l'Éducation Nationale n'assure pas la formation préparant à bon nombre de ces compétences, elle doit dispenser dans quelque 24 écoles spécialisées, parfois au profit d'un nombre très limité d'élèves, des formations initiales d'autant plus nombreuses que le maintien d'une moyenne d'âge peu élevée pour les marins entraîne un renouvellement fréquent de ces formations.

Ensuite, l'institution met en œuvre des moyens d'une telle sophistication ${ }^{5}$ qu'elle doit d'avoir un système de formation particulièrement performant. Les compétences techniques acquises dans ses métiers devenant rapidement obsolètes, elle doit en outre assurer une formation continue de ses personnels.

En raison de la durée limitée des carrières militaires, elle a aussi le souci de dispenser un enseignement théorique permettant de valoriser l'expérience acquise par l'attribution de diplômes civils : CAP, BEP, BTS, licence, diplôme d'ingénieur.

Enfin, dans un contexte de restrictions budgétaires, pour réduire les coûts de la formation, elle actionne essentiellement deux leviers : la recherche de synergies (regroupements, externalisation, partenariats avec l'Éducation Nationale, reconnaissance des acquis de l'expérience professionnelle) et la modernisation, qui passe notamment par la modularisation des $\operatorname{cours}^{6}$, par le blended-learning et par le recours à des simulateurs.

Cette problématique a incité l'institution à préconiser le recours aux TICE. Certes, les chercheurs (Chaptal, 1999, p. 243 ; Legros et Crinon, 2002, p. 9-10) s'accordent à leur dénier une efficacité per se. Mais ils ont mis en évidence leurs potentialités dans le domaine des apprentissages. Legros, Maître de Pembroke et Talbi (2002, p. 26-32) ont proposé une typologie des didacticiels en fonction des trois grands paradigmes de dont ils s'inspirent : le béhaviorisme, le cognitivisme et le constructivisme. «Technologies intellectuelles qui amplifient et modifient nombre de fonctions cognitives bumaines " (Lévy, 1997, p. 187), les TICE participent, selon Hommage (1999, 28-30), à l'acquisition des processus d'apprentissage : la métacognition, le conflit sociocognitif et la médiation. Pour Lebrun (2007, p. 123-168), elles contribuent au développement des pédagogies actives : l'apprentissage par résolution de problèmes, l'apprentissage coopératif et l'apprentissage par projet. Ce

4 Cf. : Défense et Sécurité nationale, 2008, Le Livre blanc. Paris, Odile Jacob (p. 127-228).

5 À titre d'exemple, le porte-avion Charles de Gaulle, servi par un équipage de 2800 hommes, bénéficie des innovations technologiques les plus performantes dans de nombreux domaines : la structure, la furtivité, la stabilisation, le pilotage, l'énergie et de la propulsion, le catapultage et le décollage, l'atterrissage et l'appontage, le garage et la manutention des aéronefs, le système de combat, les transmissions et les communications, la vie à bord.

6 La modularisation des cours, adaptée à l'homogénéité des formations, a été adoptée par le CIN en 2009 et mise progressivement en pratique pour tous les niveaux de formation. 
même auteur (2002, p. 77-113) a montré qu'elles favorisent l'interactivité sous ses différentes formes : il en résulte qu'elles sont performantes dans l'enseignement à distance.

La politique volontariste de recours aux TICE trouve un terrain d'application privilégié au CIN, qui est un lieu de focalisation de synergies concourant à l'actualisation de leurs potentialités, non seulement au plan organisationnel, comme nous nous proposons de le montrer dans cette étude, mais aussi au plan pédagogique (disciplines scientifiques et techniques pour l'enseignement desquelles elles ont fait la preuve de leur efficience) et au plan économique (volume et homogénéité des formations, polyvalence des personnels et des outils) (Sar, 2012, p. 245-288).

\section{Une méthodologie mixte}

Nous avons eu recours, pour la collecte des données, à l'analyse de documents officiels et à des entretiens.

Nous avons suivi l'incidence de la préconisation de l'usage des TICE en analysant les Instructions et de Directives émanant du Bureau des Écoles et de la Formation (PM/FORM) de la Direction du Personnel Militaire de la Marine (DPMM) ainsi que les Ordres Permanents (OP), les organigrammes émanant du commandant (CDT) et les Instructions Permanentes (IP) émanant du directeur de l'enseignement (DDE) du CIN.

Par la méthode des entretiens, nous avons recueilli des informations d'ordre qualitatif relatives à l'application des textes officiels auprès du Chef du Bureau de la Formation de la DPMM, auprès des Chefs de Cours et auprès des responsables des différentes instances du service TICE du CIN.

\section{Le remodelage de l'organisation de la formation}

La politique volontariste de recours aux TICE a un impact important sur l'organisation de la formation : implication des acteurs en place, installation de nouveaux acteurs, mise en œuvre de nouveaux dispositifs ${ }^{7}$.

\section{L'implication des acteurs en place}

Le Bureau des Écoles (PM/FORM) de la Direction des Personnels Militaires de la Marine (DPMM)

Cette entité, qui est responsable du pilotage des TICE, dont elle vérifie la cohérence et valide les procédures et les outils, a publié de nombreux textes concernant les objectifs généraux et opérationnels, l'organisation générale, les instances et les fonctions spécifiques créées dans la Marine Nationale ${ }^{8}$.

7 L'analyse des interactions entre les TIC et les organisations est un axe fécond de la recherche en SIC (Gramaccia, 2001 ; Durampart, 2007).

8 Cf., notamment, consultables au Bulletin officiel des armées :

- Instruction n 593/DEF/DPMM/FORM (18 décembre 2000). 


\section{$\mathrm{C} \& O \mathrm{n}^{\circ} 45$}

De ces textes, il ressort que les TICE permettent :

- de renforcer la performance de la formation en l'adaptant à la situation de chaque marin et en favorisant l'innovation pédagogique des formateurs ;

- de diminuer parfois le temps de présence en école, en cohérence avec la modularisation des formations selon les compétences;

- de répondre en partie au besoin toujours croissant de maîtrise de la complexité, notamment dans le cadre des nouvelles formations pour les équipages réduits des bâtiments de nouvelle génération ;

- de favoriser le développement des outils nécessaires à une véritable capitalisation des savoirs numériques en facilitant l'exploitation et l'archivage des ressources.

Les cadres de la formation

$\mathrm{Au} \mathrm{CIN}$, tous les responsables de l'encadrement et de l'enseignement se voient attribuer de nouvelles tâches liées au développement des TICE. Ainsi, les chefs de cours doivent gérer des formations à distance, les instructeurs peuvent être chargés du développement de simulateurs légers ${ }^{9}$, les formateurs assurent la mise en ligne de leurs cours, le développement d'Aides Pédagogiques Informatisées (API) et le tutorat à distance.

\section{L'installation de nouveaux acteurs}

\section{La Commission des TICE}

Cette instance, présidée par le chef du Bureau PM/FORM, se réunit annuellement pour examiner les questions générales et pour prononcer des arbitrages sur la production des outils.

Le Comité de coordination de simulation des écoles (CCSE)

Le CCSE a pour objectif de fédérer et de soutenir les initiatives des écoles qui développent des simulateurs. Il a pour missions d'harmoniser les processus de développement des outils, d'assurer une activité de conseil auprès des équipes de projets et de partager la veille technologique.

Il est composé des instructeurs développeurs de simulateurs opérant au sein des secteurs de simulation des écoles. Son secrétariat permanent est assuré par le Secteur Simulation du CIN.

- Instruction générale n 452 DEF/DPMM/FORM/NP (21 juillet 2006).

- Directive n ${ }^{\circ}$ 0-688-2009 DEF/DPMMM/FORM/NP (12 janvier 2009).

- Directive n ${ }^{\circ}$ 0-18187-2009 DEF/DPMM/FORM/NP, (8 avril 2009).

- Instruction n ${ }^{\circ}$ 0-29785-2010/DEF/DPMM/FORM (21 juin 2010).

9 Ils suivent un parcours de formation axé sur la maîtrise des TICE 


\section{Le Service TICE du CIN}

Cette entité regroupe : le Centre de Production Multimédia des Ecoles, le Secteur E-Learning, le Secteur Simulation, le Secteur Aides Pédagogiques Informatisées.

\section{Le Centre de Production Multimédia des Écoles (CPME)}

Le CPME assure, pour l'ensemble des écoles, un rôle de veille, d'étude et de standardisation, concernant les équipements de production et de diffusion, les procédures de conception de didacticiels, les chartes graphiques, les normes de gestion et de diffusion documentaires pour l'enseignement, la formation technique dans le domaine des TICE.

Il réalise différents types de produits : API, tutoriels, simulateurs légers, vidéogrammes.

En outre, il assure l'administration fonctionnelle du portail de formation E-Form.

\section{Le Secteur E-learning}

En 2006 a été créée au CIN la Cellule Atrium, devenue en 2010 Cellule e-learning, puis, en 2011, Secteur e-learning. Centre d'expertise pour la Marine dans le domaine de la formation à distance, cette entité a pour rôle le développement d'outils de formation à distance. Elle distribue chaque année huit dispositifs d'apprentissage à distance, à des centaines d'exemplaires, disponibles en consultation et téléchargement, notamment le CD-Rom Préparation scientifique du cours du Brevet Supérieur.

\section{Le Secteur Simulation}

Pour l'acquisition de simulateurs lourds, la Marine Nationale fait appel à l'industrie. Mais, pour les simulateurs légers, dont le développement ne rentre pas dans le cadre de la production du CPME, les écoles s'orientent vers une solution autonome. Ainsi, le CIN a créé, en 2007, le Pôle Simulation devenu depuis le Secteur Simulation du service TICE. Cette entité a pour missions de fédérer les études en matière de conception et de réalisation de simulateurs simples dédiés à l'enseignement, de coordonner l'action des principaux acteurs de la simulation, de participer aux séminaires traitant de ce domaine de compétences ainsi que d'assurer l'expertise, dans le domaine de la simulation, pour les moyens de formation.

\section{Le Secteur API}

Ce secteur est chargé de produire des didacticiels de type API et des sites web légers, de fournir un conseil et une expertise technique dans les phases de conception et de définition des didacticiels plus complexes, d'accompagner les instructeurs dans la mise en œuvre des TICE. 


\section{$\mathrm{C} \& O \mathrm{n}^{\circ} 45$}

Le Secteur Ingénierie de Formation ${ }^{10}$

Cette entité, apparue dans l'organigramme du CIN en 2012, est chargée de définir les modalités d'intégration des TICE dans la conception d'une nouvelle formation.

\section{Les Concepteurs-Intégrateurs MultiMEDIA (CIMMEDIA)}

Les CIMMEDIA, acteurs prépondérants de la chaîne de production des TICE, assurent, outre l'élaboration de didacticiels, des fonctions de formation, de conseil et de veille au sein des écoles.

Le CIN a reçu mandat de développer les synergies entre CIMMEDIA en créant sur Intramar ${ }^{11}$ un espace dédié.

Les Gestionnaires de la Documentation d'Enseignement (GEDE)

Les GEDE sont responsables de la gestion de la documentation électronique d'enseignement des écoles. Ils sont chargés de mettre en ligne les dossiers et les ressources pédagogiques multimédias sur les sites appropriés du portail de formation. Ils assurent l'archivage et la standardisation des contenus, sont responsables de l'indexation de la recherche des documents par mots-clés et administrent localement l'espace dédié à leur école sur les différents sites.

\section{La mise en œuvre de nouveaux dispositifs ${ }^{12}$}

Les principaux dispositifs TICE mis en œuvre sont les API, un CD-Rom interactif de préparation à distance, les simulateurs, le portail E-form. Nous avons entrepris par ailleurs une approche de l'évaluation de ces outils, qui tend à montrer leur efficience, aux plans pédagogique et économique (Sar, 2012, p. 296-355).

Un dispositif polyvalent : les API

Une API est un outil polyvalent. Ou bien elle est utilisée par l'enseignant pendant le cours en présentiel, ou bien l'apprenant la retrouve dans le cours mis en ligne pour le travail personnel (approfondissement et révisions, préparation d'une formation à distance via un CD-Rom). Destinées à faciliter la présentation d'un contenu d'enseignement, les API sont de différents types, selon les besoins auxquels elles répondent : la sensibilisation, l'illustration, la schématisation, la démonstration. L'API de sensibilisation a pour objectif de susciter l'intérêt de l'apprenant en présentant une activité dans son contexte, en montrant soit l'intérêt de la maîtriser, soit les risques qu'il y a à ne pas

10 Le concept d' « ingénierie de formation " (Le Boterf, 2004) est à distinguer de celui d' "ingénierie pédagogique » ( $f$. Paquette, 2005, p. 7).

11 Intramar est l'Intranet de la Marine Nationale.

12 La mise en place de nouveaux dispositifs s'accompagne, au CIN, de l'aménagement de salles médiatisées pour la diffusion des cours (avec tableau numérique interactif) ou le travail personnel et de l'utilisation d'outils synchrones, comme la visio-conférence, dans l'enseignement à distance. 
la maîtriser. À cet effet, une séquence vidéo, qui conjugue dynamisme et réalisme, est le produit le plus adéquat.

L'API d'illustration vise à favoriser l'identification et la reconnaissance par l'apprenant d'un système et de ses éléments constitutifs, en lien avec les capacités à acquérir.

L'API de schématisation est destinée à favoriser la compréhension d'un système : elle en donne une vision globale et présente d'une façon simplifiée ses éléments, en décrivant de façon synthétique leurs relations fonctionnelles.

L'API de démonstration est utilisée pour favoriser la mémorisation de la chronologie des actions : elle décompose les différentes étapes de l'activité, met en évidence les difficultés, explique comment les résoudre.

L'API de mise en situation cherche à favoriser l'acquisition de la capacité faisant l'objet de l'apprentissage dans ses trois phases : identification, compréhension, exécution. Elle doit être sécurisante et régulatrice. À cette fin, elle recourt à la correction des erreurs et à la répétition des actions jusqu'à la maîtrise.

Pour faciliter la tâche des apprenants, le CIN a défini une charte graphique s'appliquant à toutes les API.

Un dispositif de formation à distance, le CD-Rom Préparation scientifique du cours de Brevet Supérieur

Ce produit, qui en est à sa sixième édition, a été conçu pour permettre au personnel officier-marinier de six spécialités du Brevet Supérieur d'acquérir, durant une période de préparation de plusieurs mois, les connaissances scientifiques nécessaires pour suivre l'ensemble des cours généraux ou techniques dispensés en présentiel.

Composé de 36 modules médiatisés et interactifs portant sur 6 matières (mathématiques, électricité, électronique numérique, électronique analogique, physique, chimie), il propose 17 parcours différents pour répondre aux besoins des spécialités de l'aéronautique, des forces de surface et sous-marines. Après avoir effectué les exercices proposés, l'apprenant accède à l'évaluation du module. Si la moyenne n'est pas atteinte, il lui est conseillé de revoir le cours avant d'effectuer une nouvelle évaluation. Quand la moyenne est atteinte, le résultat est transmis à la direction de cours intéressée qui a ainsi, pour chaque élève, une vision d'ensemble du travail effectué.

Des dispositifs indispensables pour la formation professionnelle : les simulateurs

Les simulateurs présents au CIN sont de deux types : des simulateurs légers, à base logicielle, développés localement, et des simulateurs lourds, réalisés par des industriels, en partenariat avec la Direction Générale de l'Armement et l'État-Major de la Marine. Nous nous bornerons ici à présenter quelques exemples. 


\section{$C \& O n^{\circ} 45$}

\section{Les simulateurs légers}

Il s'agit d'applications qui sont utilisées en présentiel et à distance. Le CIN dispose de plus de 120 simulateurs de ce type. Ils sont utilisés, pour la formation en présentiel et à distance, dans des domaines très divers : détection, mise en œuvre des armes, mécanique, électrotechnique, électronique, électricité, automatisme, traitement du signal, télécommunications, mathématiques et opérations navales.

Un exemple de simulateur de ce type est SILOE (SImulateur LOgiciel d'Électronique), qui permet de s'affranchir des inconvénients rencontrés dans les séances de travaux pratiques d'électronique : perte de temps liée aux câblages à effectuer et faible diversité des montages possibles.

\section{Les simulateurs lourds}

Ils sont indispensables pour la formation des marins, comme pour celle des aviateurs, le recours à des matériels réels n'étant plus possible aujourd'hui, pour des raisons économiques.

Sont caractéristiques de ceux présents au CIN SIMON et ESTURGEON.

SIMON (Simulateur d'Intervention Maritime et Organisation Navire)

Ce simulateur est destiné à la formation des officiers qui assureront la fonction de directeur d'intervention. Il est construit sur le principe des serious games : l'apprenant, en se mettant dans la peau d'un avatar, devient acteur de son apprentissage dans le scénario d'un incendie à bord d'un bâtiment de combat. Une fonction permet à l'avatar du formateur de se déplacer de manière invisible pour se placer en observateur. La désactivation de cette fonction permet de le faire réapparaître et d'influer sur le stress des apprenants.

ESTURGEON (Ensemble de Simulation Tactique Utilisé pour la foRmation GEnérique aux Opérations Navales)

Les fonctions représentées par ce simulateur, utilisé au CIN, où il occupe treize salles, non pour l'entraînement, mais pour la formation, sont : la navigation, la veille radar, la veille sonar active, la veille de guerre électronique, la mise en œuvre d'armes, la tenue de la situation tactique locale et les échanges de données tactiques.

Il permet :

- de « geler» la situation, pour effectuer un retour au tableau blanc ;

- de « rejouer » une partie de l'exercice, afin de visualiser ce que l'apprenant aurait pu voir avec de bons réglages ;

- de surveiller les écrans des opérateurs, depuis la salle de direction, afin de comprendre les raisons qui ont pu les conduire à prendre des décisions inadéquates ;

- de faire varier des paramètres, pour permettre, par exemple, à l'apprenant d'adapter progressivement à la vitesse réelle des missiles les contremesures qu'il enclenche ;

- d'enregistrer la séquence, en vue d'un débriefing final ; 
- d'initier l'apprenant à la gestion du stress, grâce à une simulation reproduisant fidèlement l'environnement d'un combat réel.

ESTURGEON peut mettre en jeu jusqu'à dix bâtiments sur un même thème et réaliser jusqu'à trois thèmes en parallèle. Il permet de simuler les senseurs et les systèmes d'armes réels de centaines de plates-formes (bâtiments de surface, sous-marins et aéronefs civils et militaires). Il prend en compte l'environnement (météo, bathymétrie) qui influe sur les capacités de détection et d'action des bâtiments.

Une fois par an a lieu un exercice au cours duquel chacun occupe son futur poste.

\section{Un dispositif fédérateur : le portail E-Form}

Ce portail $^{13}$ est destiné à assurer quatre fonctions : l'e-formation, l'information sur les formations et les cursus des marins, les supports d'information en école et l'information sur les écoles de la Marine.

E-Form, accessible uniquement par Intramar actuellement, regroupe plusieurs sites thématiques, dont TUNG et Médiaécole.

Le site Trait d'Union Nouvelle Génération (TUNG) a pour objectifs, dans une logique d'efficacité, de rationaliser et standardiser les produits pédagogiques, d'améliorer la productivité des écoles, d'accroître la qualité des prestations pédagogiques, d'aider les forces à prendre en charge la formation complémentaire pratique, d'offrir un environnement propice au travail personnel et à la formation continue, de proposer un système informatisé susceptible de s'ouvrir ultérieurement à des intervenants extérieurs à la Marine Nationale.

Le site Médiaécole est un environnement comportant une zone publique, qui fournit des informations relatives à l'organisation d'une école donnée, et une zone privée, destinée :

- aux formateurs, pour la diffusion de cours médiatisés, la réalisation de tests d'évaluation, la « capitalisation » du fonds documentaire d'une école ;

- aux apprenants, pour l'accession aux supports de cours, la réalisation de travaux de groupes en salle informatique, l'autoévaluation à partir de tests et l'accession à une bibliothèque de ressources pédagogiques ;

- aux directeurs de cours pour la diffusion des plannings, des classements et de la documentation.

La plateforme retenue pour E-Form est Learning Content Management System (LCMS) Ilias, outil libre, utilisé par l'OTAN et l'Armée de Terre, et pressenti pour la future plateforme d'enseignement à distance interarmées.

13 Le terme « portail » est pris, dans la Marine Nationale, au sens de "plateforme internet ou intranet donnant accès à des données des ressources informationnelles regroupées au sein d'une interface unique " (Agostinelli et Miège, 2008, p. 142). 


\section{Conclusion}

La professionnalisation des armées a conduit la Marine Nationale à engager une réforme de la formation des marins, dans un contexte de restrictions budgétaires. À cette fin, elle conduit une politique volontariste de recours aux TICE. La mise en ouvre de cette politique a profondément remodelé l'organisation de la formation professionnelle dans cette institution, notamment au CIN, lieu de focalisation de synergies concourant à l'actualisation des potentialités des TICE.

Nous proposons ci-dessous une modélisation géométrique des situations pédagogiques rencontrées au CIN : il s'agit d'une pyramide évolutive, inspirée du triangle pédagogique de Houssaye (2002) et du tétraèdre d'intégration des TIC de Lombard (2007).

Cette modélisation vise à mettre en évidence le fait que les TICE (T) constituent, au même titre que l'apprenant (A), le formateur $(\mathrm{F})$, le savoir (S), un des pôles des interactions. L'apprenant (A) est placé symboliquement au sommet de la pyramide. Il se déplace progressivement vers le haut, en fonction du niveau de compétences qu'il a acquis. Par ailleurs, la pyramide est inscrite dans un ovoïde évolutif représentant l'institution (I).

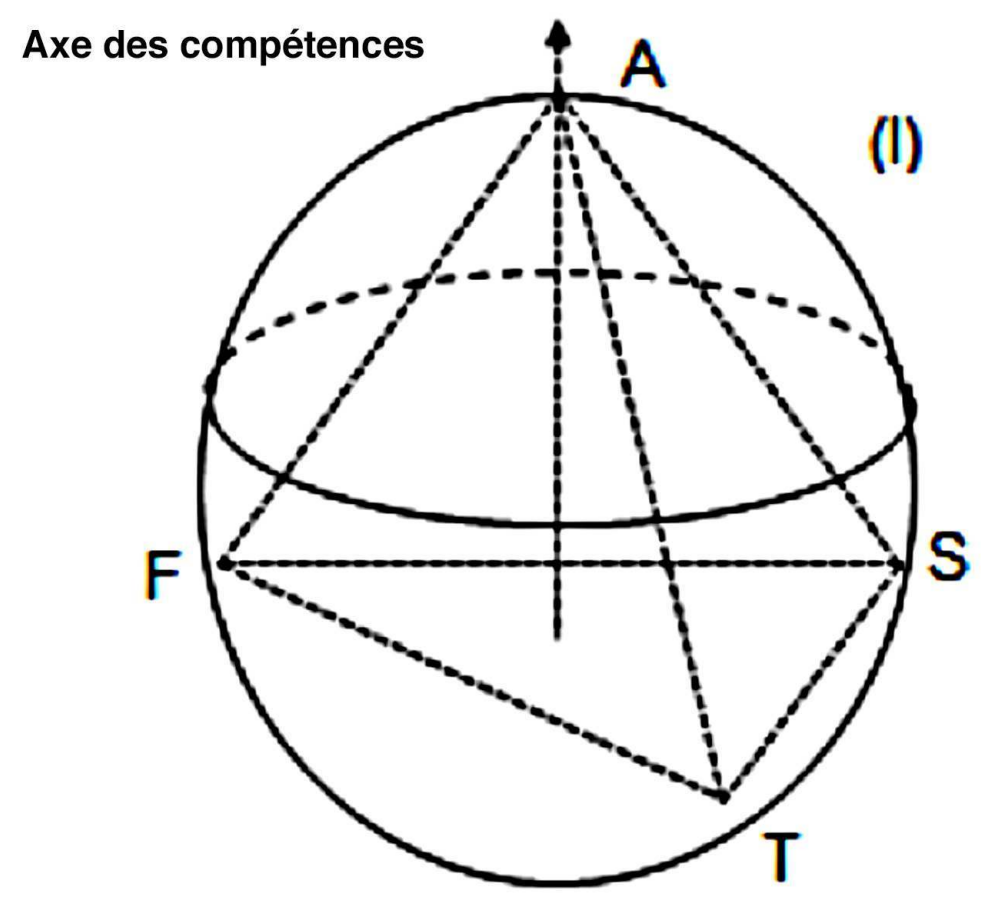

La pyramide pédagogique évolutive du CIN de Saint-Mandrier 
Quatre situations pédagogiques peuvent se présenter:

- la face AFS de la pyramide symbolise le cours ou une séance de TP en présentiel ;

- la face FST correspond à la mise en ligne par le formateur d'un cours médiatisé ;

- la face AST symbolise la formation à distance via un CD-Rom interactif, ou la révision d'un cours en salle médiatisée ;

- la face AFT symbolise le tutorat à distance.

Dans toutes ces situations, les TICE jouent un rôle. Dans le cas d'un cours ou d'une séance d'un TP en présentiel (AFS), elles sont des auxiliaires au service du formateur et de l'apprenant. Dans le cas de la mise en ligne d'un cours médiatisé (FST), c'est par le truchement des TICE que l'apprenant, absent de la situation, prend connaissance du cours. Dans le cas de l'apprentissage à distance via un CD-Rom interactif (AST), le formateur est absent de la situation mais il intervient dans la conception de l'outil et dans son contenu. Dans le cas du tutorat à distance (AFT), les échanges entre l'apprenant et le formateur se font essentiellement via les TICE (visioconférences, échanges par mails ou par téléphone).

Lomniprésence des TICE exige :

- du formateur et de l'apprenant l'acquisition de compétences dans le domaine,

- des directions de cours la gestion de la formation à distance,

- de la hiérarchie la publication de textes règlementaires, l'installation de nouveaux acteurs et la mise en œuvre de nouveaux outils.

Le remodelage de l'organisation de la formation au CIN par le recours aux TICE s'est traduit par l'implication des acteurs en place, la création de nouvelles entités, la mise en œuvre de nouveaux dispositifs.

Cette réforme étant progressive et globale, nous pensons pouvoir affirmer qu'au XXI ${ }^{\mathrm{e}}$ siècle, le modèle pédagogique du CIN, comme celui de l'École (Serres, 2011) et celui de l'Université (Bertacchini, 2010), sera marqué par le rôle des TICE.

\section{BIBLIOGRAPHIE}

AGOSTINELLI S., MIÈGE M., 2008, Espaces communs de communication des connaissances: E3C. Des espaces partagés de communication au partage des connaissances. Pessac, Presses Universitaires de Bordeaux.

ARNAUD M. (dir.), 2007, Pédagogie en ligne. Méthodes et outils. Préface de Jacques Perriault. Paris, Educaweb.

BERTACCHINI Y., 2010, "L'université au XXI ${ }^{e}$ siècle : entre tradition et métamorphose. Positionnement, mission, actions », in Actes des colloques jumelés Didactique et TICE IV et Ticemed 7, Liban, Beyrouth. 


\section{$\mathrm{C} \& O \mathrm{n}^{\circ} 45$}

CHAPTAL A., 1999, L'efficacité des technologies éducatives dans l'enseignement scolaire. Analyse critique des approches française et américaine. Thèse de doctorat en SIC, Université Paris X-Nanterre. Paris, L'Harmattan.

CHARLIER B., PERAYA D. (dirs.), 2007, Transformation des regards sur la recherche en technologie de l'éducation. Bruxelles, De Boeck Université (Coll. Perspectives en éducation \& formation).

DURAMPART M., 2007, "Les TIC et la communication des organisations : un dispositif révélateur des émergences ambivalentes de nouvelles formes organisationnelles », in Communication E Organisation, $n^{\circ}$ 31/2007, p. 115-140. [En ligne :] http:// communicationorganisation.revues.org/186 (consulté le 17.01.2014).

GRAMACCIA G., 2001, Les actes de langage dans les organisations. Paris, L'Harmattan.

HOUSSAYE J. (dir.), 2002, La Pédagogie : une encyclopédie pour aujourd'hui. (5édition). Paris, ESF.

LE BOTERF G., 2004, «L'ingénierie de la formation : Quelles définitions et quelles évolutions ? ", in Carré, P., Caspar, P. (dirs.), Traité des sciences et des techniques de la formation. Paris, Dunod, p. 365-382.

LEBRUN M., 2007, Théories et méthodes pédagogiques pour enseigner et apprendre. Quelle place pour les TIC dans l'éducation? Préface de Jean-Marie de Ketele. Bruxelles, De Boeck et Larcier (Coll. « Perspectives en éducation et formation »).

LEBRUN M., 2002, Des technologies pour, enseigner et apprendre, Bruxelles, Be Boeck Université.

LEGROS G., MAÎTRE DE PEMBROKE E., TALBI A., 2002, « Le théories de l'apprentissage et les systèmes multimédias », in Legros D., Crinon J. (Dirs.), Psychologie des apprentissages et multimédia. Paris, Armand Colin (coll. U Psychologie).

LEVY P. 1997, Cyberculture. Paris, Odile Jacob.

LOMBARD F., 2007, « Du triangle de Houssaye au tétraèdre des TIC : comprendre les interactions entre les savoirs d'expérience et ceux de recherche ", in Charlier B., Peraya D. (dirs.), Transformation des regards sur la recherche en technologie de l'éducation. Bruxelles, De Boeck Université, p. 137-154.

MIAS Ch., 2003, "Praticien-chercheur. Le problème de la double posture ", in Mesnier, P.-M., Misotte, P. (Dirs), La recherche-action, une autre manière de chercher, se former, transformer. Paris, L'Harmattan (coll. Recherche-action en pratiques sociales).

NAYMARK J. (dir), 1999, Guide du multimédia en formation. Paris, Retz.

PAQUETTE G., 2005, L’ingénierie pédagogique. Pour construire l'apprentissage en réseau. Presses de l'Université du Québec. (Coll. Des technologies pour apprendre)

SAR L., 2012, La dynamisation des potentialités des technologies de l'information et de la communication pour l'enseignement (TICE) dans la formation technique et professionnelle des marins. Analyse communicationnelle du modèle pédagogique de formation mixte du Centre d'Instruction naval (CIN) de Saint-Mandrier (Var).Thèse de doctorat en SIC, Université du Sud-Toulon Var, Laboratoire I3M.

SERRES M., 2011, Éduquer au XXI siècle. Discours prononcé dans le cadre d'une séance solennelle inter-académique sur le thème : les nouveaux défis de l'éducation, 
[En ligne :] http://www.ecoleprovence.fr/Eduquer-au-XXIe-siecle-par-Michel.html (consulté le 21.01.2014).

Résumé : La Marine Nationale, polyvalente, dotée de moyens sophistiqués servis par des personnels très spécialisés, est contrainte, dans un contexte de restrictions budgétaires, d'assurer un grand nombre de formations initiales et continues. À cette fin, elle met en œuvre, depuis la fin des années 1990, une politique volontariste de recours aux TICE. Nous nous proposons dans cette étude, relevant d'une posture en recherche-action distanciée, d'évaluer l'impact de cette politique sur l'organisation de la formation, principalement au Centre d'Instruction Naval de Saint-Mandrier (Var) : implication des acteurs en place, installation de nouveaux acteurs et mise en œuvre de nouveaux dispositifs.

Mots-clés : e-formation, formation mixte, ingénierie de formation, TICE et organisations, Marine Nationale.

Abstract : The French Navy, polyvalent and endowed with sophisticated means, served by very specialized staff, is obliged, in an historical background of financial restrictions, to offer many initial formations and in-service training. In this purpose, since the end of the nineties, it has organized a voluntary policy of having recourse to the ICTE. We intend, in this study, to value the impact of this policy on the organization of the formation, principally in the Naval training establishment of Saint-Mandrier: involvement of the existing entities and of the participants in place, installation of new entities, new participants and plans of action.

Keywords : $e$-training, mixed training, engineering of training, ICTE and organizations, French Navy. 
